\title{
Numerical Techniques for Analysis of Microbial Population with Residual Polymer in Microbial Depolymerization Process
}

\author{
Masaji Watanabe ${ }^{1}$ and Fusako Kawai ${ }^{2}$ \\ ${ }^{1}$ Graduate School of Environmental and Life Science, Okayama, University, Okayama Japan \\ ${ }^{2}$ Center for Fiber and Textile Science, Kyoto Institute Technology, Kyoto Japan
}

\begin{abstract}
This study shows that microbial consumption of carbon sources is a primary factor of microbial growth and decay in exogenous type microbial depolymerization processes. Values of residua polymer before and after cultivation of microorganisms were introduced into analysis for a microbial population. Once the microbial population was obtained, microbial depolymerization process was simulated. Those techniques were illustrated with analysis of an exogenous type depolymerization process of polyethylene glycol.
\end{abstract}

Keywords: biodegradation, xenobiotic polymer, mathematical model, inverse problem, numerical simulation

\section{Introduction}

Major industrial production of petroleum based polymers started around the mid twentieth century. Although those xenobiotic macromolecular compounds had been nonexistent until they were invented, some of them can be utilized by microorganisms for carbon sources. Those petroleum based materials have been now accumulated on the surface of the earth to potential sources of carbon dioxide emission, and now mechanism of microbial depolymerization processes must be elucidated.

Microbial depolymerization processes are classified into exogenous type processes and endogenous type processes. The primary factor of an exogenous type depolymerization process is molecular reduction by terminal liberation. Polyethylene (PE) is depolymerizable in exogenous type depolymerization processes. A mathematical model was proposed and numerical techniques were developed in studies of PE biodegradation [1]. Polyethylene glycol (PEG) is also depolymerizable in an exogenous type depolymerization process [2]. Those mathematical techniques developed for PE biodegradation were applied to a microbial depolymerization process of PEG [3]. Time dependence of degradation rate was incorporated into formulation of a PEG depolymerization process [4].

Unlike exogenous type depolymerization processes, molecules are broken down arbitrarily in endogenous type depolymerization processes. A mathematical model was proposed for simulation of an enzymatic degradation process [5]. Mathematical techniques developed for the enzymatic degradation of PVA were applied to an enzymatic hydrolysis of PLA, and degradabilities of PVA and PLA were compared [6]. Time dependence of an degradation rate was incorporated into modeling of an enzymatic hydrolysis of PLA [7]. A mathematical model proposed for endogenous type depolymerization processes was applied to exogenous type depolymerization processes of PEG [8] and PE [9]. Mathematical techniques developed for the PE biodegradation were applied to a depolymerization process of PEG [10]. Time dependence of degradability was incorporated into formulation of a PEG depolymerization process [11].

This study revisited an exogenous type microbial depolymerization process of PEG. In previous studies, weight distributions before and after cultivation of microbial consortium E-1 were introduced into inverse analyses for a molecular factor and a time factor of a degradation rate. Inverse problemes for a molecular factor and a time factor of a deradation rate were solved numerically. An initial value problem was solved numerically, and a microbial depolymerization process was simulated. In this study, a set of residual PEG before and after cultivation was introduced into an inverse analysis for the microbial population. In the following sections, mathematical model is described, techniques to solve inverse problems for the micriobial population are 
illustrated, and numerical results are presented. A numerical result for the microbial population and experimental outcomes for an optical density were compared.

\section{Exogenous Type Microbial Depolymerization Model Propose in a Previous Study}

Suppose that $w(t, M)[\mathrm{mg}]$ is the weight distribution with respect to the molecular weight $M$ at time $t$, and that $v(t)[\mathrm{mg}]$ is the total weight of polymer molecules at time $t$. The total weight $v(t)$ of the entire residual polymer at time $t$ is

$$
v(t)=\int_{0}^{\infty} w(t, M) d M
$$

Suppose that $\sigma(t)$ is the microbial population at time $t$. System of equations (3), (4) for the weight distribution and the microbial population was proposed in previous studies [10, 11, 12, 13, 14, 15].

$$
\begin{aligned}
& \frac{\partial w}{\partial t}=\sigma(t)\left[-\lambda(M) w+c(M) \int_{M}^{\infty} \lambda(K) d(K) w(t, K) d K\right], \\
& \frac{d \sigma}{d t}=k\left[1-h \frac{\sigma}{v(t)}\right] \sigma, \\
& \quad c(M)=M e^{\rho M}, \quad d(M)=\frac{e^{-\rho M}}{M\left(1-e^{-\rho M}\right)}, \quad \rho=\frac{\log 2}{L} .
\end{aligned}
$$

Parameter $L$ is the molecular weight of a monomer unit, e.g. PE: $L=28 \quad\left(\mathrm{CH}_{2} \mathrm{CH}_{2}\right)$, PEG: $L=44 \quad\left(\mathrm{CH}_{2} \mathrm{CH}_{2} \mathrm{O}\right)$. Function $\lambda(M)$ is the molecular factor of degradation rate, whereas the microbial population $\sigma(t)$ is the time factor of degradation rate.

System of equations (3), (4) is associated with initial conditions

$$
\begin{gathered}
w(0, M)=f_{0}(M), \\
\sigma(0)=\sigma_{0},
\end{gathered}
$$

where $f_{0}(M)$ and $\sigma_{0}$ are an initial weight distribution and an initial microbial population, respectively. The initial value problem (1), (2), (3), (4) is solvable for $w(t, M)$ and $\sigma(t)$ provided the function $\lambda(M)$ and values of parameters $\sigma_{0}, k$, and $h$ are prescribed. In order to specify the function $\lambda(M)$ and values of parameters $\sigma_{0}, k$, and $h$, consider change of variables from $t$ to $\tau$ defined by

The initial value problem (1), (2), (3), (4) is solvable for $w(t, M)$ and $\sigma(t)$ provided the function $\lambda(M)$ and values of parameters $\sigma_{0}, k$, and $h$ are prescribed. In order to specify the function $\lambda(M)$ and values of parameters $\sigma_{0}, k$, and $h$, consider change of variables from $t$ to $\tau$ given by

$$
\tau=\int_{0}^{t} \sigma(s) d s
$$

Denote functions $w(t, M), \sigma(t)$, and $v(t)$ by $W(\tau, M), S(\tau)$, and $v(\tau)$, respectively, for which the relation (5) between $t$ and $\tau$ holds. Equations

$$
\begin{aligned}
\frac{\partial W}{\partial \tau} & =-\lambda(M) W+c(M) \int_{M}^{\infty} \lambda(K) d(K) W(\tau, K) d K, \\
\frac{d S}{d \tau} & =k\left[1-h \frac{S}{V(\tau)}\right],
\end{aligned}
$$

are derived from the equations (3) and (4), respectively. Note that $W(\tau, M)$ is the sole unknown variable of equation (9), whereas equation (4) involves two unknowns variables $w(t, M)$ and $\sigma(t)$. Note also that

$$
V(\tau)=\int_{0}^{\infty} W(\tau, M) d M
$$


holds. Suppose that $F_{1}(M)$ is the weight distribution $W(\tau, M)$ for $\tau=T_{1}$, that is, $F_{1}(M)=W\left(T_{1}, M\right)$, and that $F_{2}(M)$ is the weight distribution $W(\tau, M)$ for $\tau=T_{2}\left(0 \leq T_{1}<T_{2}\right)$, that is, $F_{2}(M)=W\left(T_{2}, M\right)$. Given $F_{1}(M)$ and $F_{2}(M)$, equation (9), the initial condition

$$
W\left(T_{1}, M\right)=F_{1}(M)
$$

and the final condition

$$
W\left(T_{2}, M\right)=F_{2}(M)
$$

form an inverse problem for $\lambda(M)$, for which the solution of the initial value problem (6), (8) also satisfies the final condition (9). Numerical techniques for the inverse problem were developed in previous studies. Weight distributions after before cultivation of the microbial consortium E1 were assigned to the functions $F_{1}(M)$ and $F_{2}(M)$, respectively, and values of $T_{1}$ and $T_{2}$ were set, and the inverse problem (6), (8), (9) was solved numerically. Once the function $\lambda(M)$ was specified, equation (9) was solved for $W(\tau, M)$ with the initial condition

$$
W(0, M)=f_{0}(M)
$$

Given $m+1$ pairs of values of $t$ and $v(t),\left(t_{0}, v_{0}\right),\left(t_{1}, v_{1}\right), \ldots,\left(t_{m}, v_{m}\right)$, corresponding the values of $\tau, \tau_{0}, \tau_{1}, \ldots, \tau_{m}$ are obtained by solving $V\left(\tau_{i}\right)=v\left(t_{i}\right) \quad(i=1,2, \ldots, m)$.

A solution of the equation is not only a function of $\tau$, but also a function of parameters $\sigma_{0}, k$, and $h$. Suppose $S\left(\tau, \sigma_{0}, k, h\right)$ is the solution of the equation (10) with initial value $\sigma_{0}$. The change of variables (8) leads to $t=q\left(\tau, \sigma_{0}, k, h\right)$, where

$$
q\left(\tau, \sigma_{0}, k, h\right)=\int_{0}^{\tau} \frac{d r}{S\left(r, \sigma_{0}, k, h\right)}
$$

Given $m$ pairs of values of $t$ and $\tau,\left(t_{i}, \tau_{i}\right) \quad(i=1,2, \ldots, m)$, define functions $g_{i}\left(\sigma_{0}, k, h\right) \quad(i=1,2, \ldots, m)$ by

$$
g_{i}\left(\sigma_{0}, k, h\right)=q\left(\tau_{i}, \sigma_{0}, k, h\right)-t_{i},
$$

and consider the equations for $\sigma_{0}, k$, and $h$,

$$
g_{i}\left(\sigma_{0}, k, h\right)=0 \quad(i=1,2, \ldots, m) .
$$

Various methods were applied to systems of equations such as system (14). Those are the NewtonRaphson method in conjunction with the bisection method, the NewtonRaphson method in conjunction with the Newton's method, the Newton-Raphson method, and the GaussNewton method $[12-15,17,18]$.

\section{Microbial Population in Exogenous Type Microbial Depolymerization Process of PEG}

Previous study shows that the function $V(\tau)$ is well approximated with an exponential function $V(\tau)=v(0) e^{-\mu \tau}$. In this study, system of equations (12) was analyzed. The results from cultivation of the symbiotic mixed culture E-1 on PEG 6000 was reported [16]. Let $t_{0}=0, t_{1}=1, t_{2}=3, t_{3}=4, t_{4}=5, t_{5}=7$, and $t_{6}=11$. The residual PEG $v_{0}, v_{1}, \ldots, v_{6}$ at time $t_{0}, t_{1}, \ldots, t_{6}$, respectively, are given. Recall that function $S\left(\tau, \sigma_{0}, k, h\right)$ is the solution of the equation (7) with the initial value $\sigma_{0}$. The value of the parameter $\mu=2$ was set, and the GaussNewton method [19] was applied to the nonlinear least square problem for a minimizer of the expression. Table 1 sows the convergence process of a sequence of parameter values generated by the Gauss-Newton method. For those values of the parameters in the last row of the table $1, t=q\left(\tau, \sigma_{0}, k, h\right)$ was generated numerically.

Figure 1 shows the residual PEG. 
TABLE I: Convergence process of a sequence generated by the Gauss Newton method. The initial values of $\sigma_{0}, k$, and $h$ were $0.1,0.2$, and 400.0 , respectively, and the residual at the $n^{\text {th }}$ step $\sqrt{\left(\sigma_{0}^{(n)}-\sigma_{0}^{(n-1)}\right)^{2}+\left(k^{(n)}-k^{(n-1)}\right)^{2}+\left(h^{(n)}-h^{(n-1)}\right)^{2}}$ reduced to a value less than $10^{-12}$ after twenty three steps. Here $\sigma_{0}^{(n)}, k^{(n)}$, and $h^{(n)}$ are approximate values of $\sigma_{0}, k$, and $h$ at the $n^{\text {th }}$ step, respectively.

\begin{tabular}{|r|c|c|c|c|}
\hline$n$ & $\sigma_{0}{ }^{(n)}$ & $k^{(n)}$ & $h^{(n)}$ & Residual \\
\hline 0 & 0.1000000000000000 & 0.2000000000000000 & 400.0000000000000000 & \\
\hline 1 & 0.0994712200790175 & 0.2647019893267910 & 453.5594521318570000 & 53.5594912157667000 \\
\hline 2 & 0.0976723354256255 & 0.2698380666711560 & 431.3419505965090000 & 22.2175022018332000 \\
\hline 3 & 0.0990035526379217 & 0.2668439034076890 & 429.8402255085150000 & 1.5017286629299200 \\
\hline 4 & 0.0987054665615930 & 0.2683211108119130 & 430.4765200611240000 & 0.6362963371556590 \\
\hline 5 & 0.0987741465360885 & 0.2679302821343420 & 430.2985094747810000 & 0.1780110286311540 \\
\hline 6 & 0.0987589117356565 & 0.2680228992252650 & 430.3396351261650000 & 0.0411257584946348 \\
\hline 7 & 0.0987623045164953 & 0.2680019590888500 & 430.3301874992620000 & 0.0094476507183294 \\
\hline 8 & 0.0987615556325857 & 0.2680066364757630 & 430.3322933644540000 & 0.0021058705197855 \\
\hline 9 & 0.0987617208562342 & 0.2680056005562030 & 430.3318262622940000 & 0.0004671033380734 \\
\hline 10 & 0.0987616844459683 & 0.2680058293231320 & 430.3319294229440000 & 0.0001031609106899 \\
\hline 11 & 0.0987616924673173 & 0.2680057788810690 & 430.3319066724800000 & 0.0000227505216587 \\
\hline 12 & 0.0987616907005094 & 0.2680057899961860 & 430.3319116858780000 & 0.0000050134110688 \\
\hline 13 & 0.0987616910896440 & 0.2680057875476640 & 430.3319105814590000 & 0.0000011044226628 \\
\hline 14 & 0.0987616910039412 & 0.2680057880869710 & 430.3319108247190000 & 0.0000002432613418 \\
\hline 15 & 0.0987616910228162 & 0.2680057879681910 & 430.3319107711420000 & 0.0000000535778426 \\
\hline 16 & 0.0987616910186591 & 0.2680057879943510 & 430.3319107829410000 & 0.0000000117997003 \\
\hline 17 & 0.0987616910195750 & 0.2680057879885870 & 430.3319107803410000 & 0.0000000025999677 \\
\hline 18 & 0.0987616910193727 & 0.2680057879898600 & 430.3319107809150000 & 0.0000000005732105 \\
\hline 19 & 0.0987616910194175 & 0.2680057879895810 & 430.3319107807910000 & 0.0000000001233505 \\
\hline 20 & 0.0987616910194076 & 0.2680057879896410 & 430.3319107808160000 & 0.0000000000248975 \\
\hline 21 & 0.0987616910194099 & 0.2680057879896270 & 430.3319107808090000 & 0.0000000000073328 \\
\hline 22 & 0.0987616910194097 & 0.2680057879896290 & 430.3319107808110000 & 0.0000000000026148 \\
\hline 23 & 0.0987616910194097 & 0.2680057879896280 & 430.3319107808110000 & 0.0000000000005116 \\
\hline
\end{tabular}

Values of optical density $\mathrm{OD}_{610}, O_{0}, O_{1}, O_{2}, O_{3}, O_{4}, O_{5}$, and $O_{6}$ were recorded at $t_{0}=0, t_{1}=1, t_{2}=3$, $t_{3}=4, t_{4}=5, t_{5}=7$, and $t_{6}=11[16]$. Figure 3 shows points $\left(O_{0}, \sigma\left(t_{0}\right)\right),\left(O_{1}, \sigma\left(t_{1}\right)\right),\left(O_{2}, \sigma\left(t_{2}\right)\right),\left(O_{3}, \sigma\left(t_{3}\right)\right)$, $\left(O_{4}, \sigma\left(t_{4}\right)\right),\left(O_{5}, \sigma\left(t_{5}\right)\right)$, and $\left(O_{6}, \sigma\left(t_{6}\right)\right)$. A linear relation $\sigma=a O+b$ between the microbial population $\sigma$ and the optical density $O$ was assumed. Figure 2 also shows a result of the least square approximation for $a$ and $\mathrm{b}$; $a \approx 0.023357, b \approx 0.117776$ based on $\left(O_{0}, \sigma\left(t_{0}\right)\right),\left(O_{1}, \sigma\left(t_{1}\right)\right),\left(O_{2}, \sigma\left(t_{2}\right)\right),\left(O_{3}, \sigma\left(t_{3}\right)\right),\left(O_{4}, \sigma\left(t_{4}\right)\right)$, and $\left(O_{5}, \sigma\left(t_{5}\right)\right)$. Figure 4 shows the curve $(t, \sigma(t))$. It also shows conversions of $O_{0}, O_{1}, O_{2}, O_{3}, O_{4}, O_{5}$, and $O_{6}$ according to the least square approximation.

\section{Conclusion}

In previous studies $[10-15,17,18]$, weight distributions before and after cultivation of microorganisms in culture media were introduced into inverse analysis of the molecular factor and the time factor of a degradation rate. Those include the inverse problems for the molecular factor (6), (8), (9) and the time factor (12). This study demonstrates that an exogenous type microbial depolymerization process is simulated with a set of residual polymer before after cultivation of microorganisms. 


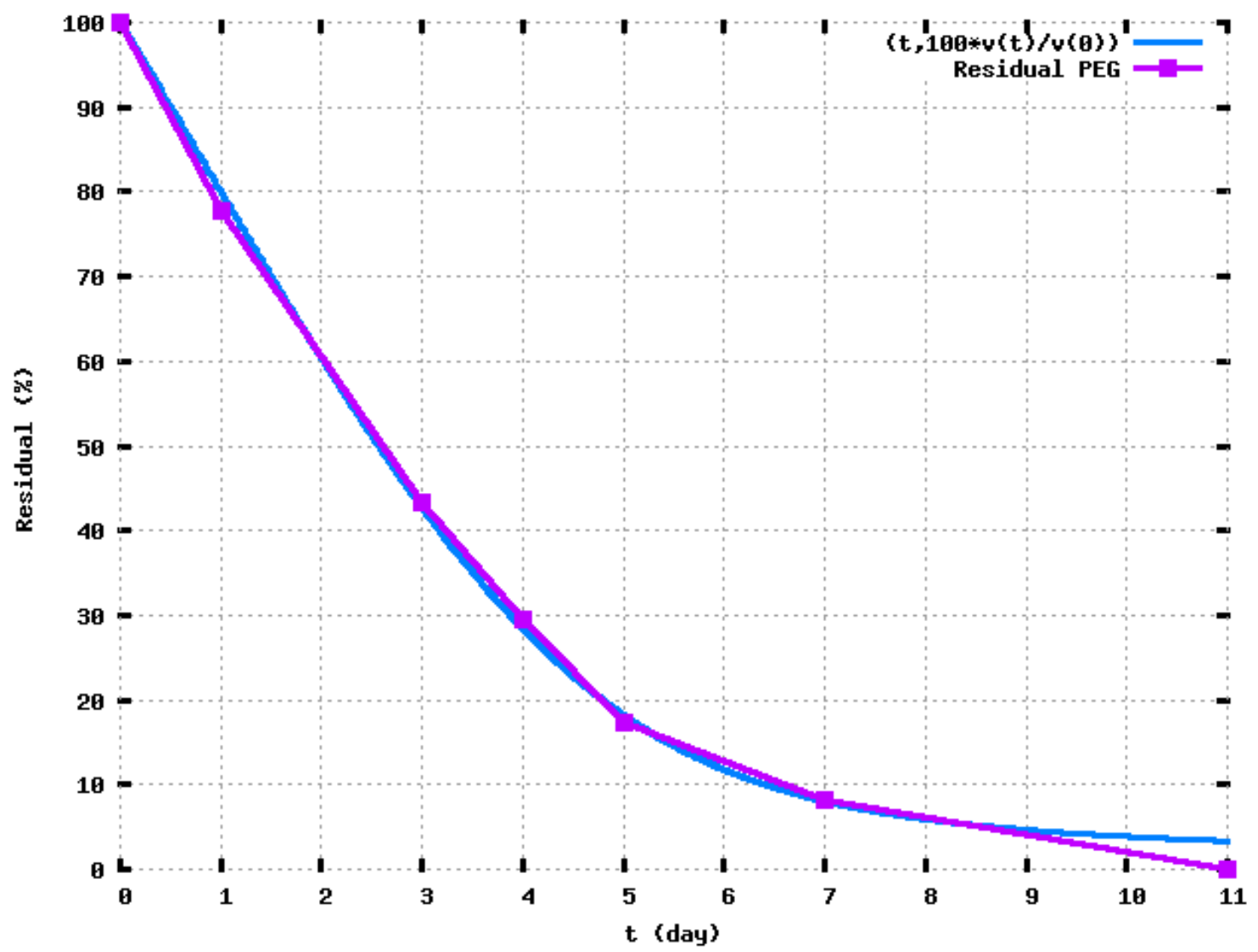

Fig. 1: Residual PEG. Curve $(t, y)=\left(q\left(\tau, \sigma_{0}, k, h\right), 100.0 \times V(\tau) / v(0)\right)$. The figure also shows Residual PEG at $t_{0}=0$, $t_{1}=1, t_{2}=3, t_{3}=4, t_{4}=5, t_{5}=7$, and $t_{6}=11$.

Figure 3 shows the transition of viable cell density whereas the optical density of culture media was an outcome from a mixture of viable cells and inviable cells. The symbiotic mixed culture E-1 consists of $S$. terrae and Rhizobium sp. The results concerning the residual PEG are outcomes from a microbial depolymerization process where $S$. terrae was a primary PEG utilizing bacteria. Figure 4 shows the transition of viable $S$. terrae whereas Rhizobium sp. as well as inviable cells were incorporated in the outcomes of the $\mathrm{OD}_{610}$. Viable cell density of S. terrae and Rhizobium sp. were measured by colony counting on nutrient agar plates in another experiment [16]. A further study is required to validate the numerical results by comparison with the experimental results. 


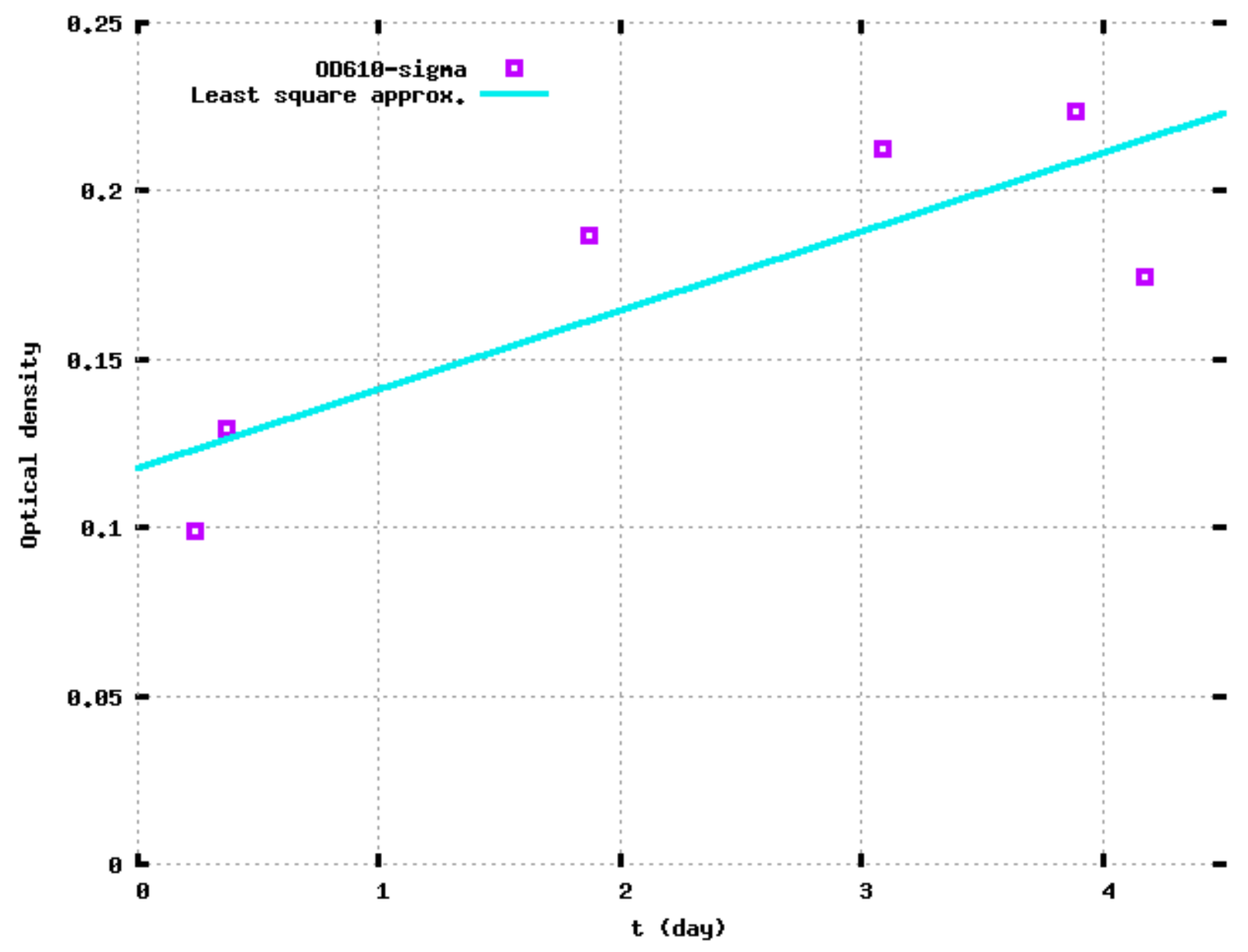

Fig. 2: $\left(O_{0}, \sigma\left(t_{0}\right)\right),\left(O_{1}, \sigma\left(t_{1}\right)\right),\left(O_{2}, \sigma\left(t_{2}\right)\right),\left(O_{3}, \sigma\left(t_{3}\right)\right),\left(O_{4}, \sigma\left(t_{4}\right)\right)$, and $\left(O_{5}, \sigma\left(t_{5}\right)\right)$. A least square approximation $\sigma=a O+b$ is also shown $(a \approx 0.023357, b \approx 0.117776)$.

\section{Acknowledgements}

The authors thank K. Yamamoto for his technical support. This work was supported by JSPS KAKENHI Grant Number 16K05276. 


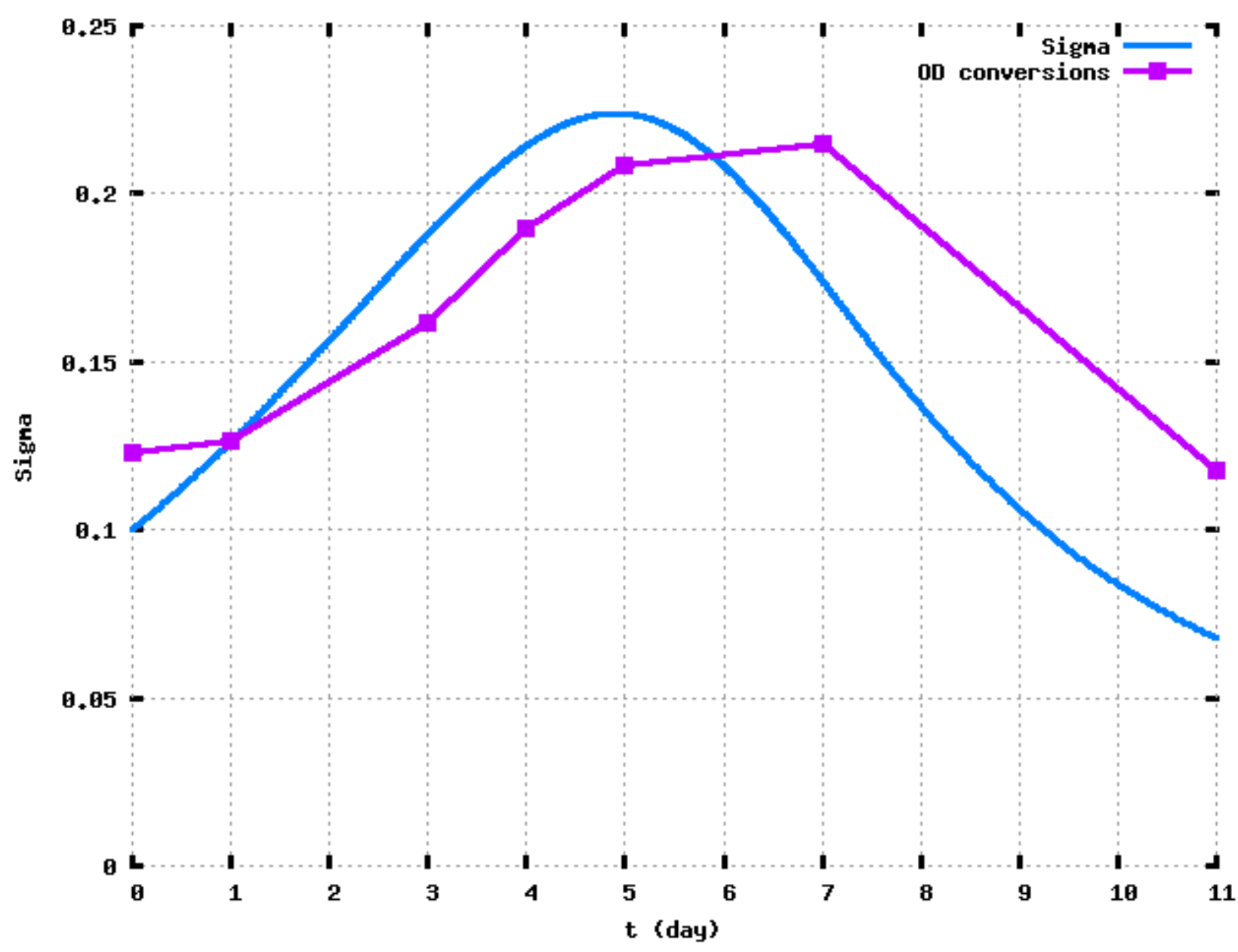

Fig. 3: Curve $(t, \sigma(t))=\left(q\left(\tau, \sigma_{0}, k, h\right), S\left(\tau, \sigma_{0}, k, h\right)\right)$ and conversions of $\mathrm{OD}_{610}$. Values of $\mathrm{OD}_{610} O_{i} \quad(i=0,1, \ldots, 6)$ were converted according to the least square approximation (Fig. 3).

\section{References}

[1] Masaji Watanabe, Fusako Kawai, Masaru Shibata, Shigeo Yokoyama, Yasuhiro Sudate, Shizue Hayashi, Analytical and computational techniques for exogenous depolymerization of xenobiotic polymers, Mathematical Biosciences 192 (2004) 19-37.

https://doi.org/10.1016/j.mbs.2004.06.006

[2] Fusako Kawai, Takuhei Kimura, Masahiro Fukaya, Yoshiki Tani, Koichi Ogata, Tamio Ueno, Hiroshi Fukami, Bacterial oxidation of polyethylene glycol, Applied and Environmental Microbiology, Vol. 35, No. 4, 679--684, 1978.

[3] M. Watanabe, F. Kawai, Numerical simulation of microbial depolymerization process of exogenous type, Proc. of 12th Computational Techniques and Applications Conference, CTAC-2004, Melbourne, Australia in September 2004, Editors: Rob May and A. J. Roberts, ANZIAM J. 46(E) pp.C1188--C1204, 2005. http://journal.austms.org.au/ojs/index.php/ANZIAMJ/article/view/1014

[4] M. Watanabe and F. Kawai, Effects of microbial population in degradation process of xenobiotic polymers, In P. Howlett, M. Nelson, and A. J. Roberts, editors, Proceedings of the 9th Biennial Engineering Mathematics and Applications Conference, EMAC-2009, volume 51 of ANZIAM J., pages C682--C696, September 2010.

http://journal.austms.org.au/ojs/index.php/ANZIAMJ/article/view/2433

[5] Masaji Watanabe, Fusako Kawai, Mathematical modelling and computational analysis of enzymatic degradation of xenobiotic polymers, Applied Mathematical Modelling 30 (2006) 1497-1514

https://doi.org/10.1016/j.apm.2005.12.011 
[6] Masaji Watanabe, Fusako Kawai, Sadao Tsuboi, Shogo Nakatsu, Hitomi Ohara, Study on Enzymatic Hydrolysis of Polylactic Acid by Endogenous Depolymerization Model, Macromolecular Theory and Simulations 16 (2007) 619626. https://doi.org/10.1002/mats.200700015

[7] M. Watanabe, F. Kawai, Modeling and analysis of biodegradation of xenobiotic polymers based on experimental results, Proceedings of the 8th Biennial Engineering Mathematics and Applications Conference, EMAC-2007, Editors: G. N. Mercer and A. J. Roberts, ANZIAM J. 49 (EMAC-2007) pp.C457--C474, March 2008. http://journal.austms.org.au/ojs/index.php/ANZIAMJ/article/view/361

[8] M. Watanabe and F. Kawai, Study of biodegradation of xenobiotic polymers with change of microbial population, In W. McLean and A. J. Roberts, editors, Proceedings of the 15th Biennial Computational Techniques and Applications Conference, CTAC-2010, volume 52 of ANZIAM J., ages C410--C429, July 2011. http://journal.austms.org.au/ojs/index.php/ANZIAMJ/article/view/3965

[9] Watanabe M, Kawai F (2012) Modeling Biodegradation of Polyethylene with Memoryless Behavior of Metabolic Consumption, J Bioremed Biodegrad 3: 146.

[10] M. Watanabe and F. Kawai, Study on microbial depolymerization processes of xenobiotic polymers with mathematical modelling and numerical simulation, In Mark Nelson, Mary Coupland, Harvinder Sidhu, Tara Hamilton, and A. J. Roberts, editors, Proceedings of the 10th Biennial Engineering Mathematics and Applications Conference, EMAC-2011, volume 53 of ANZIAM J., pages C203--C217, June 2012. http://journal.austms.org.au/ojs/index.php/ANZIAMJ/article/view/5107

[11] Masaji Watanabe, Fusako Kawai, Simulation for microbial depolymerization processes of polyethylene glycol, Proceedings of the 2013 International Conference on Advances in Intelligent Systems in Bioinformatics, Editors: Dr. Ford Lumban Gaol, Dr. Benfino Soewito, Prof. Dr. Mohamed Bououdina, Prof. Dr. Mu-Song Chen, intel-13, Advances in Intelligent Systems Research, Atlantis Press, February 2014, 71 - 76. ISBN: 978-94-6252-000-4, ISSN: 1951-6851 http://www.atlantis-press.com/php/pub.php?publication=intel-13

[12] Masaji Watanabe, Fusako Kawai, Numerical Techniques for Inverse Problems from Modeling of Microbial Depolymerization Processes, International Journal of Applied Engineering Research ISSN 0973-4562 Volume 11, Number 8 (2016) pp 5461-5468. http://www.ripublication.com/ijaer16/ijaerv11n8_18.pdf

[13] Masaji Watanabe, Fusako Kawai, Modeling microbial depolymerization process of exogenous type with consumption rate and microbial growth, Submitted.

[14] Masaji WATANABE and Fusako KAWAI, Simulation of Microbial Depolymerization Process with Exponential Consumption of Carbon Source, 2016 International Conference on Computer, Mechatronics and Elelctronic Engineering (CMEE 2016), DEStech Publications, Inc., Lancaster, Pennsylvania, U.S.A., 2016, 426-431, ISBN: 978-1-60595-406-6

[15] Masaji Watanabe, Fusako Kawai, Numerical Techniques for Simulation of Microbial Depolymerization Process with Time Factor of Degradation Rate, Submitted.

[16] FUSAKO KAWAI, SHOGO ENOKIBARA, Symbiotic Degradation of Polyethylene Glycol (PEG) 20,000-Phthalate Polyester by Phthalate Ester- and PEG 20,000-Utilizing Bacteria, Journal of Fermentation and Bioengineering, Vol. 82, No. 6, 575-579, 1996. https://doi.org/10.1016/S0922-338X(97)81255-X

[17] Masaji Watanabe, Fusako Kawai, Numerical Study of Microbial Depolymerization Process with the NewtonRaphson Method and the Newton's Method, International Journal of Engineering Research \& Science (IJOER), Vol2, Issue-2, February- 2016, Pages 119-130. ISSN: [2395-6992] http://ijoer.com/Paper-February-2016/IJOER-JAN-2016-55.pdf

[18] Masaji Watanabe, Fusako KawaI, Numerical Study of Inverse Problems in Modeling and Simulation of Microbial Depolymerization Processes, Volume 1, 2016, International Journal of Chemistry and Chemical Engineering Systems, 2016, 46-55. ISSN: 2367-9042 http://www.iaras.org/iaras/filedownloads/ijcces/2016/016-0006.pdf

[19] S. Gratton, A. S. Lawless, and N. K. Nichols, Approximate Gauss-Newton Method for Nonlinear Least Squares Problems, SIAM Journal of Optimization, 18(1), 2007, 106-132 https://doi.org/10.1137/050624935 